\title{
Instantaneous Normal Mode Analysis of Supercooled Water
}

\author{
Emilia La Nave ${ }^{1}$, Antonio Scala ${ }^{1}$, Francis W. Starr ${ }^{1 *}$, \\ Francesco Sciortino ${ }^{2}$, and H. Eugene Stanley ${ }^{1}$ \\ ${ }^{1}$ Center for Polymer Studies, Center for Computational Science, and Department of Physics, Boston University, Boston, MA \\ 02215 USA \\ ${ }^{2}$ Dipartmento di Fisica e Istituto Nazionale per la Fisica della Materia, Universitá di Roma "La Sapienza", Piazzale Aldo \\ Moro 2, I-00185, Roma, Italy
}

(September 12, 2018)

We use the instantaneous normal mode approach to provide a description of the local curvature of the potential energy surface of a model for water. We focus on the region of the phase diagram in which the dynamics may be described by the mode-coupling theory. We find, surprisingly, that the diffusion constant depends mainly on the fraction of directions in configuration space connecting different local minima, supporting the conjecture that the dynamics are controlled by the geometric properties of configuration space. Furthermore, we find an unexpected relation between the number of basins accessed in equilibrium and the connectivity between them.

PACS numbers: 61.43.Fs, 64.70.Pf, 66.10.Cb

The study of the dynamics in supercooled liquids has received great interest in recent years [1] due to possibilities opened by novel experimental techniques [2], detailed theoretical predictions [3], and by the possibility of following the microscopic dynamics via computer simulation 细. One important theoretical approach developed recently is the ideal mode coupling theory (MCT) [3], which quantitatively predicts the time evolution of correlation functions and the dependence on temperature $T$ of characteristic correlation times. Unfortunately, the temperature region in which $\mathrm{MCT}$ is able to make predictions for the long time dynamics is limited to weakly supercooled states [5]. In parallel with the development of MCT, theoretical work [6 9] has called attention to thermodynamic approaches to the glass transition, and to the role of configurational entropy in the slowing down of dynamics. These theories, which build on ideas put forward some time ago 10 12], stress the relevance of the topology of the potential energy surface (PES) explored in supercooled states. Detailed studies of the PES may provide insights on the slow dynamics of liquids, and new ideas for extending the present theories to the deep supercooling regime.

\footnotetext{
${ }^{*}$ Current Address: Polymers Division and Center for Theoretical and Computational Materials Science, National Institute of Standards and Technology, Gaithersburg, Maryland 20899
}

The instantaneous normal mode (INM) approach uses the eigenvectors (normal modes) and eigenvalues of the Hessian matrix (the second derivative of the potential energy) to provide an "instantaneous" representation of the PES in the neighborhood of an equilibrium phase space point. Examples of INM analysis have appeared for a few representative liquids 13 16]. In this Letter we present INM calculations for a rigid water molecule model, the SPC/E potential [17], for several temperatures and densities in supercooled states. The reasons for selecting such a system are twofold: (i) The SPC/E model has been studied in detail, and it has been shown that its dynamics follows closely the predictions of MCT 18,19]. Furthermore, the $(\rho, T)$ dependence of the configurational entropy has been calculated, and shown to correlate with the dynamical behavior [20]. (ii) The SPC/E model has a maximum of the diffusion constant $D$ under pressure along isotherms, as observed experimentally [21]. The maxima in $D$ can be used as sensitive probes for testing proposed relations between the change in topology of configuration space and the change in $D$.

We analyze recent simulations 19] for six densities between 0.95 and $1.3 \mathrm{~g} / \mathrm{cm}^{3}$ and five temperatures for each density. For each state point, we extract 100 equallyspaced configurations [22], from which we calculate and diagonalize the Hessian using the center of mass and the three principal inertia momenta vectors as molecular coordinates 23. For each configuration, we classify the imaginary modes into two categories: (i) shoulder modes and (ii) double-well modes, according to the shape of the PES along the corresponding eigenvector [24]. The shoulder modes are modes for which the negative curvature appears as a result of a local anharmonicity; the double-well modes include only the directions in configuration space which connect two adjacent minima. The classification of the modes is done by studying the shape of the PES along the eigenvector corresponding to the mode, i.e. beyond the point in configuration space where such modes have been calculated. In this respect, the potential energy along the eigenvector direction may be different from the actual curvilinear direction described by the time evolution of the eigenvector under consideration. Furthermore, some modes classified as double-well may be related to intra-basin motions [25] and therefore may not be relevant to describe diffusivity. In the present 
study we show - using the $D$ maxima line as a guide that these spurious features are negligible.

Fig. 1 shows the density dependence of the fractions of imaginary (unstable) modes $f_{u}$, double-well modes $f_{d w}$, and of shoulder modes $f_{s h}$, where $f_{u}=f_{d w}+f_{s h}$; we also show $D$ for the same state points. The close correlation of $f_{d w}$ and $D$ is striking. At $\rho \approx 1.15 \mathrm{~g} / \mathrm{cm}^{3}, D$ has a maximum; at this $\rho$, the increase of diffusion constant caused by the the progressive disruption of the hydrogen bond network on increasing the density is balanced by slowing down of the dynamics due to increased packing. At the same density, $f_{u}$ and $f_{d w}$ also show maxima, supporting the view that these quantities are a good indicator of the molecular mobility. There is also a weak maximum in $f_{s h}$, but at a $\rho<1.15 \mathrm{~g} / \mathrm{cm}^{3}$ 26]. The presence of maxima in both $D$ and $f_{d w}$ at the same density suggests that, for the SPC/E potential, $f_{d w}$ is directly related to $D$. The relation between $D$ and $f_{d w}$ is shown in Fig. 2 for all the studied isochores. We note that $D$ in this system is a monotonic function of $f_{d w}$ and that all points fall on the same master curve [27], notwithstanding the large range of $D$ values analysed. Thus, surprisingly, in $\mathrm{SPC} / \mathrm{E}$ water, the knowledge of the fraction of directions in configuration space leading to a different basin, $f_{d w}$, is sufficient to determine the value of $D$. [28] We also note from Fig. 2 that $D$ vanishes, but at a small non-zero value of $f_{d w} \approx 0.007$ (vertical arrow) [29], suggesting that indeed a small number of spurious double-well modes, related to intrabasin motion [25,30], are still included in our classification.

The reduction of mobility on cooling in the studied $(\rho, T)$ range - where MCT provides a description of the dynamics - appears related to the geometrical properties of the PES, i.e. the system mobility is reduced because the number of directions connecting different local minima (needed to explore freely the configuration space) is decreasing [31, 32]. Hence, the observed reduced mobility is mainly related to the geometry of the PES, i.e. it is "entropic" in origin.

MCT seems to be able to describe the entropic slowing down of the dynamics associated with the vanishing of $f_{d w}$. This is consistent with the general consensus that the missing decay channels for the correlations responsible for the failure of MCT at very low $T$ are activated processes. To make closer contact with MCT, we compare in Fig. 3 the density dependence of the MCT critical temperature $T_{M C T}$ 19] with the $T$ at which the fraction of double-well modes goes to zero, as well as with the $T$ at which $f_{d w}$ reaches the estimated asymptotic value of 0.007 [30], to take into account the small over counting intrinsic in the mode classification. We observe that the $f_{d w}=0$ locus tracks closely the $T_{M C T}$ line, but that the $f_{d w}=0.007$ locus nearly coincides with the $T_{M C T}$ line.

The results presented here suggest that the liquid dynamics in the MCT region of SPC/E water is controlled by the average connectivity in configuration space. On the other hand, for the same model, the $(\rho, T)$ dependence of the configurational entropy $S_{\text {conf }}$ - which, in the Stillinger-Weber 10 formalism, can be defined as the logarithm of the number of different basins $\Omega$ in configuration space - has also been calculated and shown to correlate with $D$ [20]. Since no a priori relation is expected between the connectivity of the local minima and their number, we consider particularly interesting the observation of such a relation. For this reason, we show in Fig. 1 a "parametric plot" (in the parameter $D$ ) of $f_{d w}$ versus $S_{\text {conf }}$ for all studied densities. Fig. 1 shows a linear relation between $\ln \left(f_{d w}\right)$ and $S_{\text {conf }}$, i.e. between $f_{d w}$ and the number of basins (since $\Omega=\exp \left(S_{\text {conf }} / k_{B}\right)$ ) and highlight the existence of an unexpected relation between the number of basins accessed in equilibrium and the connectivity between them. This novel feature of the PES contributes to a better understanding of the water dynamics, and may contribute to the understanding of the dynamics of glass-forming liquids [33].

We thank T. Keyes for useful discussions, and the NSF for support; FS acknowledges partial support from MURST (PRIN 98).

[1] For a recent review see P. G. Debenedetti, Metastable Liquids (Princeton Univ. Press, Princeton, 1997).

[2] See for example F. Sette, M. H. Krish, C. Masciovecchio, G. Ruocco, and G. Monaco, Science 280, 1550 (1998) and P. Lunkenheimer, A. Pimenov and A. Loidl, Phys. Rev. Lett.78, 2995 (1997).

[3] W. Götze, J. Phys.: Cond. Matter, 11, A1-A45 (1999). and references therein; W. Götze, in Liquids, Freezing and Glass Transition, ed. J.P. Hansen, D. Levesque and J. Zinn-Justin, (North-Holland, Amsterdam, 1991).

[4] See for example K. Binder et al., in Complex Behavior of Glassy Systems, M. Rubí and C. Perez-Vicente Eds. (Springer, Berlin, 1997) and W. Kob, J. Phys. Cond. Matter 11, R85 (1999).

[5] We call "weakly supercooled states" the temperature range where the diffusivity has decreased 3 or 4 orders of magnitude compared to its normal liquid value $\left(\sim 10^{-5} \mathrm{~cm}^{2} / \mathrm{s}\right)$.

[6] M. Mézard and G. Parisi, J.Phys. Condens. Matter, 11, A157 (1999) and references therein.

[7] R. Speedy, J. Chem. Phys., 110, 54559 (1999); R. Speedy, J. Chem. Phys. B 103 , 4060 (1999).

[8] M.Shultz, Phys. Rev. B 57, 11319 (1998).

[9] D.C. Wallace, Phys.Rev. E 56, 4179 (1997).

[10] F.H. Stillinger and T.A. Weber, Phys. Rev. A 25, 978 (1982); Science 225, 983 (1984); F. H. Stillinger, Science 267, 1935 (1995). R. A. Laviolette and F.H. Stillinger, J.Chem.Phys. 83, 4079 (1985).

[11] R.O. Davies and G.O. Jones, Adv. in Physics 2, 370 (1953). 
[12] M. Goldstein, J. Chem. Phys. 51, 3728 (1969).

[13] T.Keyes, J. Phys. Chem. A 101, 2921 (1997); W. Li and T.Keyes , J. Chem. Phys. 111328 (1999)

[14] M. C. Riberio and P. A. Madden, J. Chem. Phys. 106, 8616 (1997); ibid. 108, 3256 (1998).

[15] M. Cho, G.R. Fleming, S. Saito, I. Ohmine and R. Stratt, J. Chem. Phys. 100, 6672 (1994).

[16] F. Sciortino and P. Tartaglia, Phys. Rev. Lett. 78, 2385 (1997).

[17] H. J. C. Berendsen, J. R. Grigera and T. P. Straatsma, J. Phys. Chem. 91, 6269 (1987).

[18] L. Fabbian, A. Latz, R. Schilling, F. Sciortino, P. Tartaglia, C. Theis, Phys. Rev. E, in press.

[19] F.W. Starr, S. Harrington, F. Sciortino, and H.E. Stanley, Phys. Rev. Lett., 82, 3629 (1999);

[20] A. Scala, F.W. Starr, E. La Nave, F. Sciortino, H.E. Stanley, cond-mat/9908301 (1999).

[21] F.X. Prielmeier, E.W. Lang, R.J. Speedy, and H.-D. L üdemann, Phys. Rev. Lett. 59, 1128 (1987); Ber. Bunsenges. Phys. Chem. 92, 1111 (1988).

[22] The configurations we use span a time of at least 25 times the relaxation time of the density-density correlation function, for two independent systems. Hence, we do not expect that insufficient sampling should skew our results.

[23] Ref. 34 has called attention to the fact that in molecular systems, the frequency distribution and the eingvectors are dependent on the coordinate system chosen. We refer the interested reader to ref. [34] for more detailed discussions on this point.

[24] S. Bembenek and B. Laird, Phys. Rev. Lett. 74, 936 (1995).

[25] J. D. Gezelter, E. Rabani, B. J. Berne J. Chem. Phys.107, 4618 (1997); S.D. Bembenek and B.B. Laird J. Chem. Phys. 104, 5199 (1996).

[26] Thus far, we cannot distinguish if the shift in the maximum of $f_{s h}$ is connected to a change of the anharmonicity as a function of density, or to spurious leakage of doublewell modes into shoulder modes in the classification procedure.

[27] We note that for $D \gtrsim 0.3 \times 10^{-6} \mathrm{~cm}^{2} / \mathrm{s}$, the relation between $D$ and $f_{d w}$ is approximately linear, in agreement with the finding of Keyes and coworkers for different liquids 13,35 .

[28] The observed relation $D\left(f_{d w}\right)$ is material dependent. Studies of the INM spectrum in different materials have shown that $D$ may correlate with $f_{u}$ or with the product $\omega_{u} f_{u}$, where $w_{u}$ is defined as the average frequency of the $f_{u}$ distribution 13,14 .

[29] The approximate value 0.007 has been obtained by fitting $D$ vs $f_{d w}$ with a cubic polynomia and evaluating the value at which $D=0.0$.

[30] Ref. 25] has shown that some of the eigenvector of the double well type may indeed point, in both directions, to the same local minimum; an example is the Mexican hat surface.

[31] A connection between glassy dynamics and the mechanism of "reduction of free directions" was proposed in R. Botet, I. A. Campbell, J. M. Flesselles and R. Jullien, in Universalities in Condensed Matter, R. Jullien, L. Peliti, R. Rammal and N. Boccara, eds. (Springer Verlag,
Berlin, 1987) p. 250. In the context of spin models, it was shown that the stretched exponential behavior observed in the long time decay of spin-spin correlation functions can be explained with the same mechanism.

[32] Such scenario is similar to the one studied in for $p$-spin models in detail. See, e.g., A. Cavagna, I. Giardina, G. Parisi, Phys. Rev. B 57, 1125 (1998)

[33] For a preliminary attempt of connecting $f_{d w}$ with $S_{\text {conf }}$ see U. Zürchen and T. Keyes, Phys. Rev. E, 602065 (1999).

[34] R.L.Murry, J.Fourkas, W.Li and T.Keyes, J. Chem. Phys. 110, 10410 (1999); ibid. 1101423 (1999).

[35] T. Keyes J. Chem. Phys. 101, 5081 (1994). 

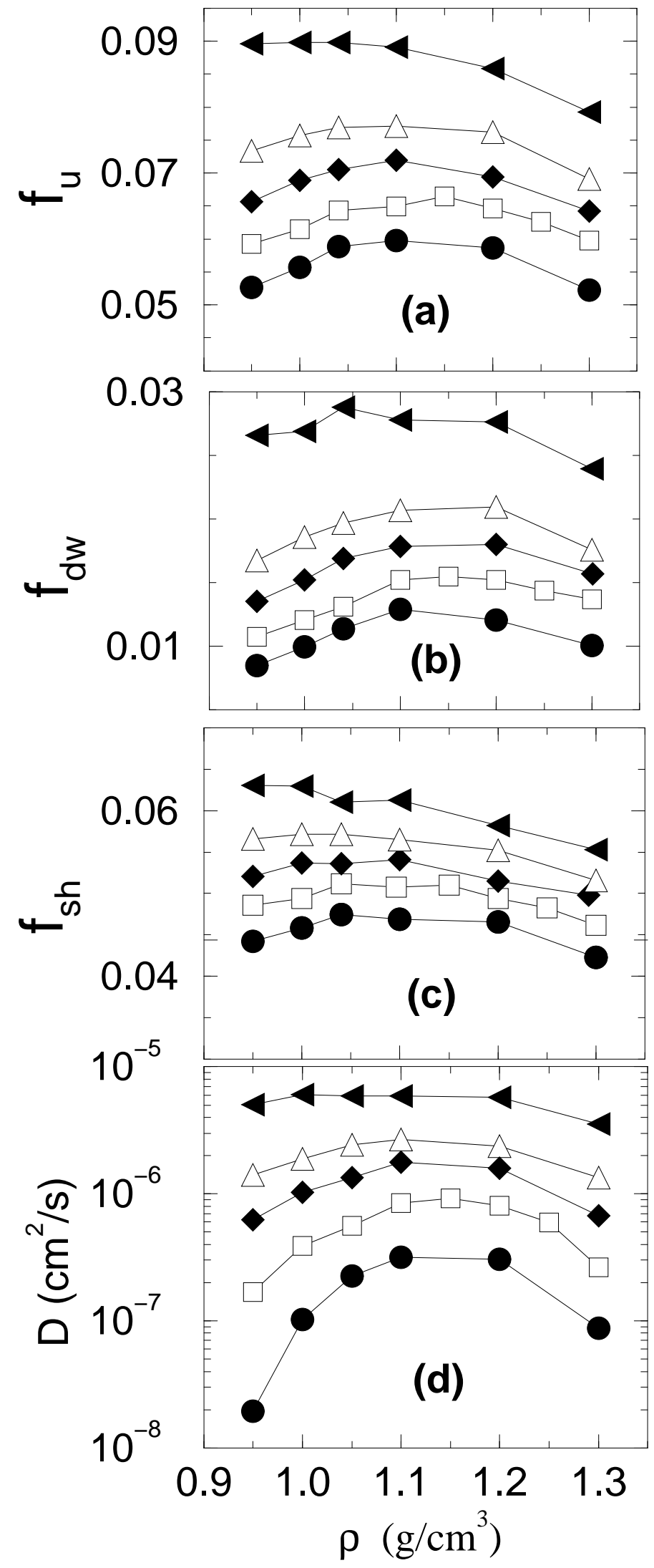

FIG. 1. Density dependence along the studied isotherms of (a) $f_{u}$, (b) $f_{d w}$, (c) $f_{s h}$. Temperatures are $T=210 K$ (circles), $T=220 K$ (squares), $T=230 K$ (diamonds) and $T=240 K$ (triangles). ${ }^{-}$(d) shows the $\rho$ dependence of $D$ for the same isotherms 19].

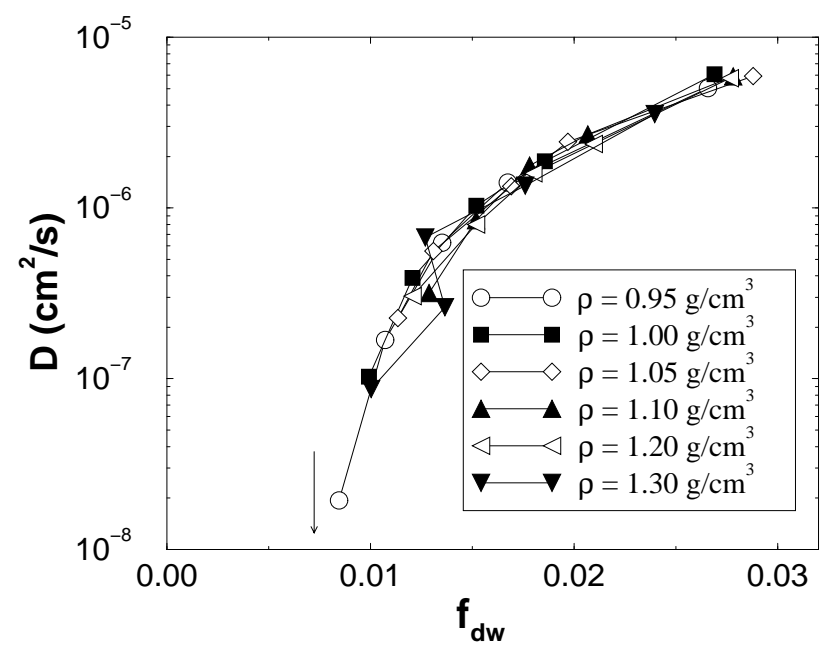

FIG. 2. Diffusion constant $D$ versus $f_{d w}$ for different isochores. The arrow indicates the value $f_{d w} \approx 0.007$, to highlight the presence - even when $D$ approaches zero - of a small number of spurious double-well modes, related to intrabasin motion 25, 30.

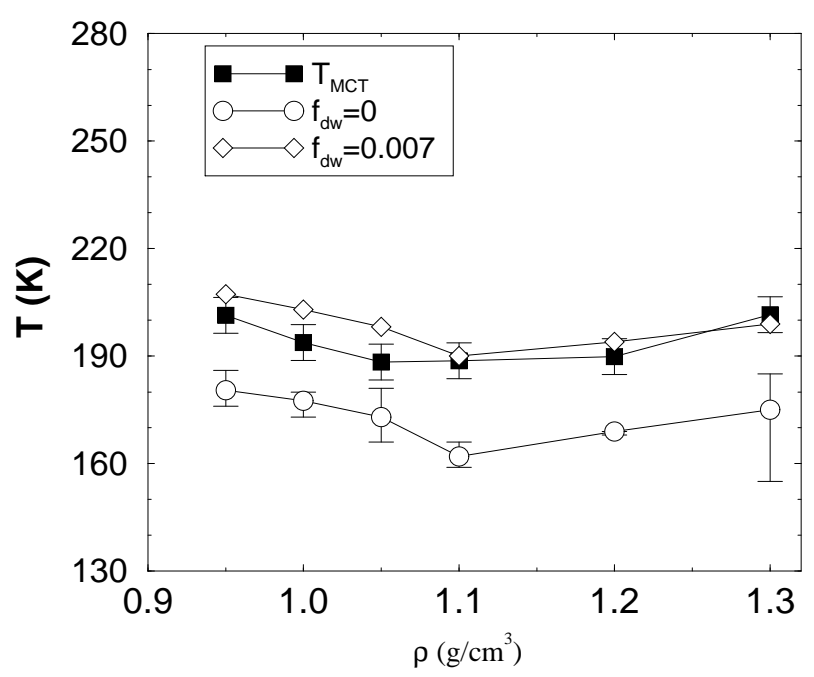

FIG. 3. Comparison of the mode coupling critical temperature $T_{M C T}$ 19, of the $f_{d w}=0$ locus and of the $f_{d w}=0.007$ locus. 


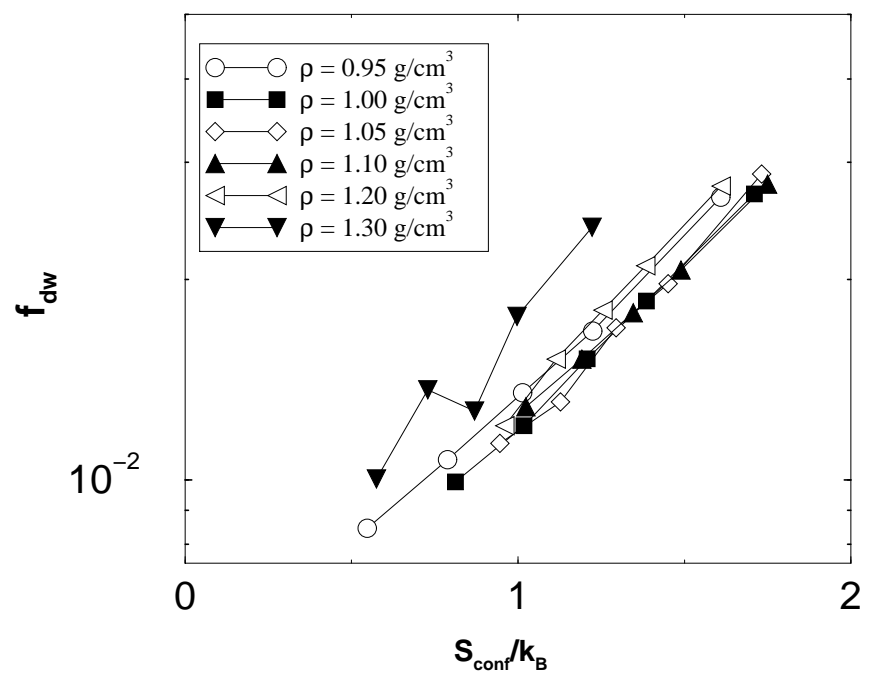

FIG. 4. Linear-log plot of $S_{\text {conf }}$ (from Ref. 20]) vs $f_{d w}$. 